\title{
A Stochastic Programming Approach for Multi-Period Portfolio Optimization
}

\author{
1st Narela Bajram ${ }^{\mathrm{a}}$, 2nd Mehmet Can ${ }^{\mathrm{b}}$ \\ ${ }^{\text {a }}$ Faculty of Business and Administration, International University of Sarajevo, \\ Hrasnicka Cesta 15, Ilidza, 71200 Sarajevo BiH, narela@ius.edu.ba \\ ${ }^{\mathrm{b}}$ Faculty of Engineering and Natural Sciences, International University of Sarajevo, \\ Hrasnicka Cesta 15, Ilidza, 71200 Sarajevo BiH, mcan@ius.edu.ba
}

\section{ABSTRACT}

\begin{abstract}
An Asset-Liability Management model with a novel strategy for controlling risk of underfunding is presented in this paper. The basic model involves Multi-Period decisions (portfolio optimization) and deals with the usual uncertainty of investment returns and future liabilities. Therefore, is it well suited to a stochastic programming approach.

We consider the problem of rebalancing policy to accomplish some investment's criteria. Transaction costs have also been a subject of concern in this paper. In particular, a large amount of transactions usually make asset price move in an unfavorable direction. Therefore, the first problem neglects transactions cost while the second does not.
\end{abstract}

Keywords: Stochastic Linear Programming (SLP), Portfolio Optimizations, Asset- Liability Management (ALM) , Scenario Trees. 


\section{INTRODUCTION}

The Asset-Liability Management (ALM) problem has crucial importance to pension funds, insurance companies and banks where business involves large amount of liquidity. Indeed, the financial institutions apply ALM to guarantee their liabilities while pursuing profit. The liabilities may take different forms: pensions paid to the members of the scheme in a pension fund, savers' deposits paid back in a bank, or benefits paid to insurers in the insurance company. A common feature of these problems is the uncertainty of liabilities and the resulting risk of underfunding. The other major uncertainty originates from asset returns. Together they constitute a nontrivial difficulty in how to manage risk in the model applied by the financial institution. The need for multi-period planning additionally complicates the problem.

Stochastic programming provides a general purposemodeling framework, which captures the real-world features such as turnover constraints, transaction costs, risk aversion, limits on groups of assets and other consideration. However, the optimization model turns out to be intractable for the enormous number of decision variables, especially for the multi-stage problems.

One of the first industrially applied models of this type was the stochastic linear program with simple recourse developed by Kusy and Ziemba in 1986. This model captured certain characteristics of ALM problems: it maximized revenues for the bank in the objective under legal, policy, liquidity, cash flow and budget constraints to make sure that deposit liability is met as much as possible. Under computational limits at the time when it was developed, this model took the advantage of stochastic linear programming so as to be practical even for the large problems faced in banks.

In this paper we demonstrate how ALM model can be applied for asset allocation in financial markets. We assume a very simple model with two "assets"; stocks and bonds.

In a real world the trading of financial assets comes at a cost. Stockbrokers and other financial intermediaries charge their customers a fee when selling and buying financial assets. Therefore, the first problem neglects transaction cost while the second does not.

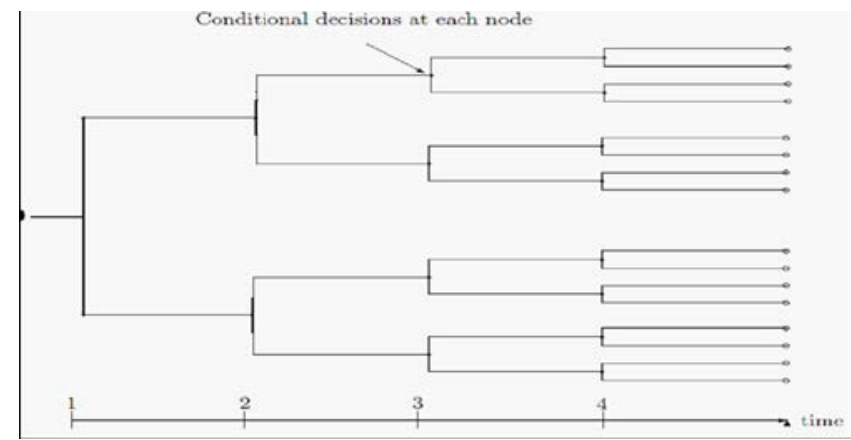

Figure 1: A scenario tree for a multi-stage stochastic program
Moreover, it is important for decision makers to rebalance the portfolio during the investment period as they may wish to adjust the asset allocations according to updated information on the market. The strategy which is currently optimal may not be optimal any more as the situation changes. Thus it is important to reconsider the strategy and make the necessary change in order to remain in the optimal position. Taking this into account, the problem is multiperiod and at the beginning of each period, new decisions are made. Such a multi-stage ALM model allows different decisions through the investing process.

To make it easier to model, we consider the problem stage by stage and with portfolio rebalancing done at the beginning of each stage. Also, the uncertainties of asset returns are implemented with discrete distributions, in which case an event tree is used to capture the uncertainties in multiple stages throughout the whole decision process, e.g. as shown in Figure 1.The nodes at each stage represent possible future events. Asset returns, liabilities and cash deposits are subject to uncertain future evolution. Meanwhile, the asset rebalancing is done after knowing which value the asset returns and liabilities take at each node.

The paper is organized as follows: in Section 2 we provide a classification of life-cycle asset allocation models based on the type of available solutions. Section 3 describes the stochastic programming model, in particular the formulation of the objective, the optimization approach for its linearization, and the generation of scenarios.

\section{Overview OF ASSET AlLOCATION MODELS}

The classical treatments of strategic asset allocation can be traced back to Samuelson (1969) and Merton (1969, 1971). In the light of Markowitz' seminal papers on single-period portfolio selection, the early literature focused on conditions leading to the optimality of myopic policies, i.e., conditions under which portfolio decisions for multi-period problems coincide with those for single period problems. In addition, the lack of computing power lead to formulate models driven by the quest for closed form solutions. To achieve these objectives, rather restrictive assumptions were made, and many of these models' results turned out to be inconsistent with conventional wisdom as expressed by the so-called Samuelson puzzle: whereas one of the main results from early multi-period portfolio models is that the fractions of risky assets are constant over time, this contradicts the advice obtained from many professionals in practice that investors should hold a share of risky assets which declines steadily as they approach retirement (often called the age effect). Since then, many researchers have tried to resolve this puzzle which is mainly rooted in some of the (simplifying) assumptions used in early models (fixed planning horizon, time-constant investment opportunities, no intermediate consumption, etc.). 
Research in the area of life-cycle asset allocation models regained momentum in the early 1990s for two main reasons: first, a number of economic factors increased the number of people with sizeable wealth to invest (the "generation of heirs"), coupled with increased uncertainty about the security of public pension systems. Second, the enormous increase in computer power enabled the solution of models with more realistic assumptions. A number of additional features have been added to the classical models, in many cases with the goal of resolving the Samuelson puzzle: stochastic labor income, time-varying investment opportunities, parameter uncertainty (with and without learning), special treatment of certain asset classes (real estate), and habit formation, to name just the most important developments.

In contrast to other approaches in the literature using nonlinear optimization (see, e.g., Blomvall and Lindberg 2002; Gondzio and Grothey 2007), we use multi-period stochastic linear programming (SLP) to solve the problem of optimal life-cycle asset allocation and consumption. This method has been explicitly chosen with the practical application of the approach in mind. Many features which are considered important for investment decisions in practice can be easily incorporated when using SLP. For example, personal characteristics of the investor can be taken into account (e.g., mortality risk, risk attitude, retirement, future cash flows for major purchases or associated with other life events). Combined with the availability of efficient solvers, this explains why the SLP approach has been successfully applied to a wide range of problems (see, e.g., Wallace and Ziemba 2005). To nest classical analytical results from this area within our model, we maximize expected utility of consumption over the investor's lifetime and expected utility of bequest rather than other objectives which can be implemented more easily (e.g., piecewise linear or quadratic penalty functions, or minimizing $\mathrm{CVaR}$ ).

An important reference for the present paper is Campbell et al. (2003). They model asset returns and state variables as a first-order vector autoregression $\operatorname{VAR}(1)$ and consider Epstein-Zin utility with an infinite planning horizon. Additional assumptions include the absence of borrowing and short-sale constraints. Linearizing the portfolio return, the budget constraint, and the Euler equation, they arrive at a system of linear-quadratic equations for portfolio weights and consumption as functions of state variables. This system of equations can be solved analytically, yielding solutions which are exact only for a special case (very short time intervals and elasticity of intertemporal substitution equal to one), and accurate approximations in its neighbourhood.

The SLP used in the present paper has been applied successfully to a number of related problems. To cite only a few examples, there are applications in insurance (Cariño and Ziemba 1994, 1998; Cariño et al. 1998), and the pension fund industry (e.g., Gondzio and Kouwenberg 2001). Zenios (1999) surveys large-scale applications of SLP to fixed income portfolio management. General aspects of applying such models in a strategic asset allocation context are discussed in Ziemba and Mulvey (1998)), Pflug and Swietanowski (2000), Gondzio and Kouwenberg (2001), Wallace and Ziemba (2005), and Geyer and Ziemba (2007). Particular aspects that are relevant in a life-cycle portfolio context are discussed in Geyer et al. (2007).

\section{MODEL DESCRIPTIONS}

Asset-liability management (ALM) models assist financial institutions in decision making on asset allocations considering full use of fund and resources available. The model aims to maximize the overall revenue, sometimes as well as revenue at intermediate stages, with restrictions of risk. Risk in the ALM problems is present in two aspects: a possible loss of investment and missing the ability to meet liabilities. The returns of assets and the liabilities are both uncertain. It is essential in ALM modelling to deal with uncertainties as well as with risks. Stochastic programming approach is naturally applicable to problems which involve uncertainties.

\subsection{A two-stage model: asset- liability management}

The best way to introduce multistage stochastic models is a simple asset liability management (ALM) model. We have an initial wealth W0, that should be properly invested in such a way to meet a liability $\mathrm{L}$ at the end of the planning horizon $\mathrm{H}$.

If possible, we would like to own a terminal wealth $\mathrm{WH}$ larger than L; however, we should account properly for risk aversion, since there could be some chance to end up with a terminal wealth that is not sufficient to pay for the liability, in which case we will have to borrow some money.

A nonlinear, strictly concave utility function of the difference between the terminal wealth $\mathrm{WH}$, which is a random variable, and the liability $\mathrm{L}$ would do the job, but this would lead to a nonlinear programming model.

As an alternative, we may build a piecewise linear utility function like the one illustrated in Fig. 1

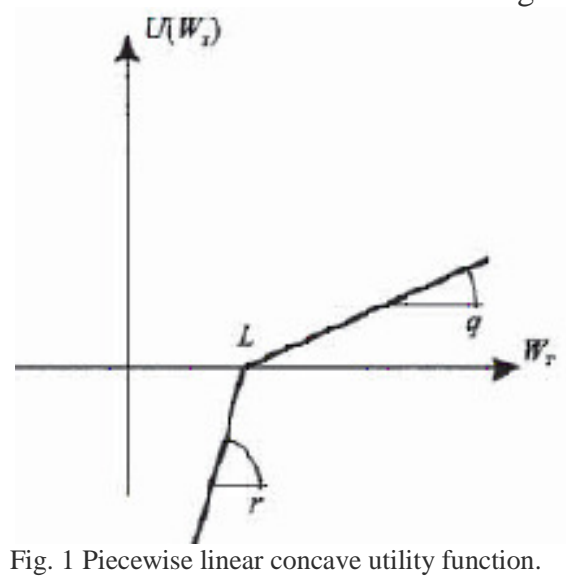

The utility is zero when the terminal wealth $\mathrm{WH}$ matches the liability exactly. 
If the slope $r$ penalizing the shortfall is larger than $q$, this function is concave (but not strictly).

The portfolio consists of a set of 2 assets. For simplicity, we assume that we may rebalance it only at a discrete set of time instants $\mathrm{t}=1, \ldots, \mathrm{H}-1$, with no transaction cost; the initial portfolio is chosen at time $t=0$, and the liability must be paid at time $\mathrm{H}$.

Time period $t$ is the period between time instants $t-1$ and $t$. In order to represent uncertainty, we may build a tree like that in Fig. 2. , which is a generalization of the two-stage tree. Each node $n_{k}$ in the tree corresponds to an event, where we should make some decision. We have an initial node n0 corresponding to time $\mathrm{t}=0$.

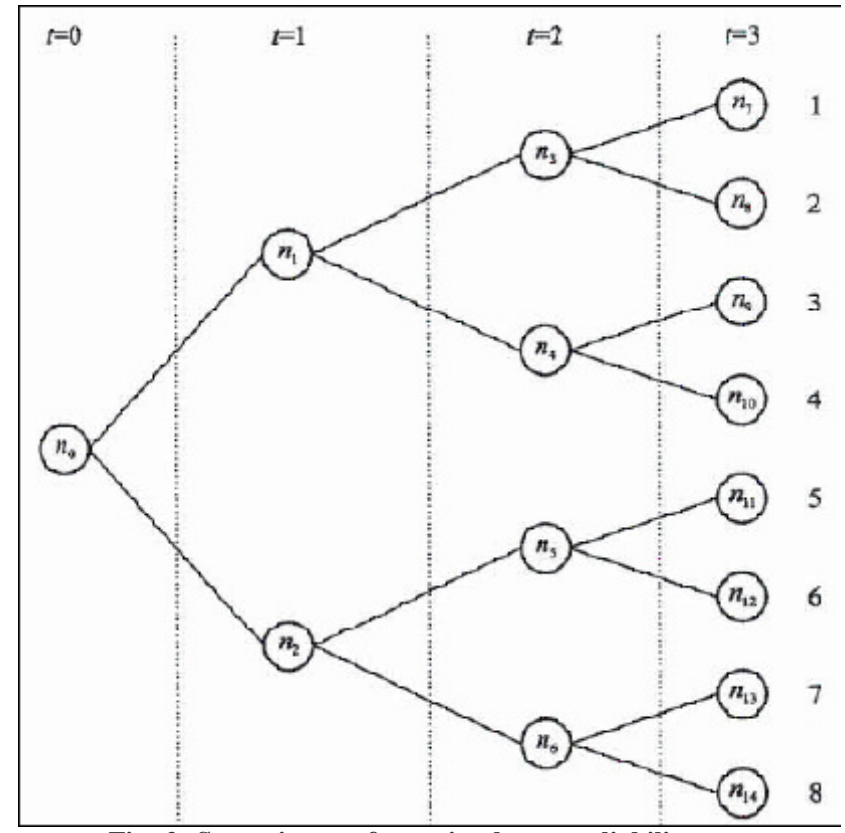
problem.

Fig. 2. Scenario tree for a simple asset- liability management

Then, for each event node, we have two branches; each branch is labeled by a conditional probability of occurrence, $\mathrm{P}(\mathrm{nk} \mid \mathrm{ni})$, where $\mathrm{ni}=\mathrm{a}(\mathrm{nk})$ is the immediate predecessor of node nk. Here, we have two nodes at time $t=1$ and four at time $t=2$, where we may rebalance our portfolio on the basis of the previous asset returns.

Finally, in the eight nodes corresponding to $t=3$, the leaves of the tree, we just compare the terminal wealth with the liability and evaluate the utility function.

Each node of the tree is associated with the set of asset returns during the corresponding time period.

A scenario consists of an event sequence, i.e., a sequence of nodes in the tree, along with the associated asset returns.

We have 8 scenarios in Fig. 2.

For instance, scenario 2 consists of the node sequence (n0, nl, n3, n8).

The probability of each scenario depends on the conditional probability of each node on its path.

If each branch at each node is equiprobable, i.e., the conditional probabilities are always $1 / 2$, each scenario in the figure has probability ps $=1 / 8$, for $s=1, \ldots, 8$. The branching factor may be arbitrary in principle; the more branches we use, the better our ability to model uncertainty; unfortunately, the number of nodes grows exponentially with the number of stages, as well as the computational effort.

At each node in the tree, we must make a set of decisions. In practice, we are interested in the decisions that must be implemented here and now, i.e., those corresponding to the first node of the tree; the other (recourse) decision variables are instrumental to the aim of devising a robust plan, but they are not implemented in practice, as the multistage model is solved on a rolling-horizon basis. This suggests that, in order to model the uncertainty as accurately as possible with a. limited computational effort, a possible idea is to branch many paths from the initial node, and less from the subsequent nodes. Each decision at each stage may depend on the information gathered so far, but not on the future; this requirement is called a. nonanticipativity condition. Essentially, this means that decisions made at time $\mathrm{t}$ must be the same for scenarios that cannot be distinguished at time $\mathrm{t}$.

To build a model ensuring that the decision process makes sense, we can associate decision variables with nodes in the scenario trees and write the model in a way that relates each node to its predecessors.

Let us now introduce the following numerical data:

- The initial wealth is 55 .

- The target liability is 80 .

- There are two assets, say, stocks and bonds; hence, I = 2.

- In the scenario tree of Fig. 2. we have up- and downbranches; in the (lucky) upbranches, total return is 1.25 for stocks and 1.14 for bonds; in the (bad) downbranches, total return is 1.06 for stocks and 1.12 for bonds. We see that bonds play the role of safer assets here. We also see that returns are a sequence of i.i.d. random variables, but more realistic scenarios can be defined.

- The reward rate $\mathrm{q}$ for excess wealth above the target liability is 1.

- The penalty rate $\mathrm{r}$ for the shortfall below the target liability is 4 .

The problem taken in this paper is a example given by $\mathrm{P}$. Brandimarte (2011) in Quantitative Methods: An Introduction for Business Management. We formulated the problem using the Wolfram Mathematical Programming System.

\subsubsection{Variables, Objective and Constrains}

The elementary building block of any objective function is a measure of wealth at $\mathrm{T}$, the last time step at which investment decision are made.

The following notation and decision variables are used in the model formulation:

- $\mathrm{N}$ is the set of event nodes; in our case

$\mathrm{N}=\{\mathrm{n} 0, \mathrm{n} 1, \mathrm{n} 2, \ldots, \mathrm{n} 14\}$ 
- Each node $n \in \mathrm{N}$, apart from the root node n0, has a unique direct predecessor node, denoted by $a(n)$ : for instance, $a(n 3)=n$

- There is a set $\mathrm{S} \cap \mathrm{N}$ of leaf (terminal) nodes; in our case

$\mathrm{S}=\{\mathrm{n} 7, \ldots, \mathrm{n} 14\}$

- For each node $\mathrm{s} \in \mathrm{S}$ we have surplus and shortfall variables ws+ and ws_, related to the difference between terminal wealth and liability.

- There is a set $\mathrm{T} \cap \mathrm{N}$ of intermediate nodes, where portfolio rebalancing may occur after the initial allocation in node no; in our case

$\mathrm{T}=\{\mathrm{n} 1, \mathrm{n} 2, \ldots, \mathrm{n} 6\}$

- For each node $\mathrm{n} \in\{\mathrm{n} 0\} \mathrm{U}$ T there is a decision variable $\mathrm{x}_{\mathrm{in}}$, expressing the money invested in asset $\mathrm{i}$ at node $\mathrm{n}$.

With this notation, the model may be written as follows:

$$
\begin{array}{rlr}
\max & \sum_{s \in S} \pi^{s}\left(q w_{+}^{s}-r w_{-}^{s}\right) & \\
\text { s.t. } & \sum_{i=1}^{I} x_{i, n_{0}}=W_{0} & \\
& \sum_{i=1}^{I} R_{1, n} x_{i, a(n)}=\sum_{i=1}^{I} x_{i n}, & \forall n \in T \\
& \sum_{i=1}^{I} R_{i s} x_{i, a(s)}=L+w_{+}^{s}-w_{-}^{s}, & \forall s \in S \\
& x_{i n}, w_{+,}^{s}, w_{-}^{s} \geq 0 &
\end{array}
$$

where Rin is the total return for asset $\mathrm{i}$ during the period that leads to node $n$, and $\pi^{\mathrm{s}}$ is the probability of reaching the terminal node $\mathrm{s} \in \mathrm{S}$; this probability is the product of all the conditional probabilities on the path that leads from root node no to leaf node s.

\subsubsection{Numerical Results}

This is an LP model that may be easily solved by the simplex algorithm, resulting in the solution of Table 1 . We may notice that in the last period the portfolio is not diversified, since the whole wealth is allocated to one asset, and we should wonder if this makes sense. Actually, it is a consequence of two features of this toy model:

- We are approximating a nonlinear utility function by a piecewise linear function, and t his may imply "local" risk neutrality, so that we only care about expected return; we should use either a nonlinear programming model or a more accurate representation of utility with more linear pieces.
Table 1. Investment strategy for a simple ALM problem.

\begin{tabular}{|l|l|l|}
\hline Node & Stocks & Bonds \\
\hline n0 & 44.793 & 13.5207 \\
\hline $\mathrm{n} 1$ & & \\
\hline $\mathrm{n} 2$ & 65.0946 & 2.16814 \\
\hline $\mathrm{n} 3$ & 36.7432 & 22.368 \\
\hline $\mathrm{n} 4$ & 83.8399 & 0 \\
\hline $\mathrm{n} 5$ & 0 & 74.286 \\
\hline $\mathrm{n} 6$ & 0 & 74.286 \\
\hline
\end{tabular}

- The scenario tree has a very low branching factor, and this does not represent uncertainty accurately.

However, the portfolio allocation in the last time period is not necessarily a critical output of the model: the real stuff is the initial portfolio allocation. As we pointed out, the decision variables for future stages have the purpose of avoiding a myopic policy, but they are not meant to be implemented.

\subsection{A two-stage model: asset- liability management with transaction cost}

The stochastic programming approach can efficiently solve the most general models, where transaction costs need to be considered, and the returns distributions have general serial dependency.

The assumptions and the limitations behind this extended model are the following:

- We are given a set of initial holdings for each asset; this is a more realistic assumption, since we should use the model to rebalance the portfolio periodically, according to a rolling-horizon strategy.

- We take proportional (linear) transaction costs into account; the transaction cost is a percentage c of the traded value, for both buying and selling an asset.

- We want to maximize the expected utility of the terminal wealth.

- There is a stream of uncertain liabilities that we have to meet.

- We do not consider the possibility of borrowing money; we assume that all of the available wealth at each rebalancing period is invested in the available assets; actually, the possibility of investing in a risk-free asset is implicit in the model.

- We do not consider the possibility of investing new cash at each rebalancing date (as would be the case, e.g. , for a pension fund).

Some of the limitations of the model may easily be relaxed. The important point we make is that when transaction costs are involved, we have to introduce new decision variables to express the amount of assets (number of shares, not the monetary value) held, sold, and bought at each rebalancing date. 


\subsubsection{Variables}

We now introduce our notation and key variables which are similar to that used in the previous ALM formulation. I is the number of assets the investor can choose from. $t$ denotes stages (points in time) and runs from $\mathrm{t}=0$ (now) to $\mathrm{t}=\mathrm{H}$.

The following (decision) variables are used in the model formulation:

$\mathrm{x}_{\mathrm{i}}^{\mathrm{n}} \geq 0\left(\mathrm{t}=1, \ldots, \mathrm{H}^{-}-1 ; i=1, \ldots, I\right) \ldots .$. the amount of asset $\mathrm{i}$ we hold at node $\mathrm{n}$

$\mathrm{z}_{\mathrm{i}}^{\mathrm{n}} \geq 0 \quad(\mathrm{t}=1, \ldots, \mathrm{H}-1 ; i=1, \ldots, I) \ldots .$. the amount of asset i purchased at node $n$.

$\mathrm{y}_{\mathrm{i}}^{\mathrm{n}} \geq 0 \quad(\mathrm{t}=1, \ldots, \mathrm{H}-1 ; i=1, \ldots, I) \ldots . .$. the amount of asset $\mathrm{i}$ sold at node $\mathrm{n}$.

$\mathrm{W}^{\mathrm{s}} \geq 0 \quad(\mathrm{~s}=1, \ldots . .8)$. terminal node $\mathrm{s} \in \mathrm{S}$.

And the notations are:

- $\mathrm{N}$ is the set of nodes in the tree; $\mathrm{n} 0$ is the root node.

- The (unique) predecessor of node $\mathrm{n} \mathrm{CN} \backslash\{\mathrm{n} 0\}$ is denoted by $\mathrm{a}(\mathrm{n})$; the set of terminal nodes is denoted by $\mathrm{S}$; as in the previous formulation, each of these nodes corresponds to a scenario, which is the sequence of event nodes along the unique path leading from $\mathrm{n} 0$ to $\mathrm{s} \mathrm{S}$, with probability $\pi_{\mathrm{s}}$.

- $\mathrm{T}=\mathrm{N} \backslash(\{\mathrm{n} 0\}$

- $\mathrm{L}^{\mathrm{n}}$ is the liability we have to meet in node $\mathrm{n} \in \mathrm{N}$; liabilities are node dependent and stochastic.

- $\mathrm{C}$ is the percentage transaction cost.

- $\mathrm{h}_{\mathrm{i}}^{\mathrm{n} 0}$ is the initial holding for asset $\mathrm{i}=1, \ldots, \mathrm{I}$ at the root node.

- $\mathrm{P}_{\mathrm{i}}{ }^{\mathrm{n}}$ is the price for asset $\mathrm{i}$ at node $\mathrm{n}$.

- $z_{i}{ }^{n}$ is the amount of asset $i$ purchased at node $n$.

- $y_{i}{ }^{n}$ is the amount of asset $i$ sold at node $n$.

- $\mathrm{x}_{\mathrm{i}}{ }^{\mathrm{n}}$ is the amount of asset $\mathrm{i}$ we hold at node $\mathrm{n}$, after rebalancing.

- $\mathrm{W}^{\mathrm{S}}$ is the wealth at terminal node s $\mathrm{E} \mathrm{S}$.

- $\mathrm{u}(\mathrm{w})$ is the utility for wealth $\mathrm{w}$; this function is used to express utility of terminal wealth.

\subsubsection{Objective and Constraints}

Taking in consideration the notation in Sec.3.2.1, we may write the following model:

$$
\begin{aligned}
& \max \sum_{s \in S} \pi^{s} v\left(W^{s}\right) \\
& \text { s.t. } x_{i}^{n a}=\vec{h}_{i}^{n_{1}}+\bar{z}_{i}^{a_{0}}-y_{i}^{n_{0}} \text {, } \\
& x_{i}^{n}=z_{i}^{n(n)}+z_{i}^{n}-y_{i}^{2}, \\
& \text { पi. (13.28-30) } \\
& (1-c) \sum_{i=1}^{I} P_{i}^{n} y_{i}^{n}-(1+c) \sum_{i=1}^{f} P_{i}^{n} z_{i}^{0}=L^{*}, \quad \forall n \in \mathcal{T} \cup\left\{n_{0}\right\} \\
& W^{s}=\sum_{i=1}^{1} P_{i}^{4} x_{i}^{a(0)}-L^{\prime}, \\
& \forall_{s} \in S \\
& x_{i}^{n}, z_{i}^{n}, y_{i}^{n}, W^{*} \geq 0
\end{aligned}
$$

The objective (13.28) is to maximize the expected utility of wealth at $\mathrm{H}$ (terminal wealth). This is a generic objective function.

Equation (13.29) expresses the initial asset balance, taking the current holdings into account; the asset balance at intermediate trading dates is taken into account by Eq. (13.30).

Equation (13.31) ensures that enough cash is generated by selling assets in order to meet the liabilities; we may also reinvest the proceeds of what we sell in new asset holdings; note how the transaction costs are expressed for selling and purchasing. Equation (13.32) is used to evaluate terminal wealth at leaf nodes; note here that we have not taken into account the need to sell assets in order to generate the cash required by the last liability; but this would make only sense if the whole fund is liquidated at the end of the planning horizon.

\subsubsection{Numerical Results}

Do the costs really have an influence on the major rebalancing at the end of each year?

If we compare the allocation of the money in Table 2 and the Table 1 from Sec.3.1.2, we see that the expected rebalancing of the investment portfolio is actually

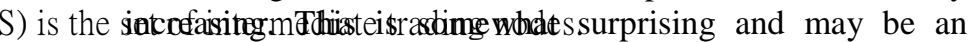
indication that transaction costs are no the most likely explanation for why investors are not performing a major rebalancing of their portfolio at the end/beginning of each year. However, this is a case for small transaction costs. The expected rebalancing of the investment decreases when increasing the proportional transaction cost. (Fleten, SteinErik and Lindset, Snorre 2007).

Table 2 Investment strategy for a simple ALM problem

\begin{tabular}{|l|l|l|}
\hline Node & Stocks & Bonds \\
\hline n0 & 4851.74 & 0 \\
\hline n1 & 0 & 2580.56 \\
\hline n2 & 5273.62 & 0 \\
\hline n3 & 0 & 3082.32 \\
\hline n4 & 0 & 3061.96 \\
\hline n5 & 0 & 3028.52 \\
\hline n6 & 6563.65 & 0 \\
\hline
\end{tabular}

The most important point in our model is that we have assumed that the liabilities must be met. This may be a very hard constraint; if extreme scenarios are included in the formulation, as they should be, it may well be the case that the model above is infeasible. Therefore, the formulation should be relaxed in a sensible way; we could consider the possibility of borrowing cash; we could also introduce suitable penalties for not meeting the liabilities.

In principle, we could also require that the probability of not meeting the liabilities is small enough; this leads to chanceconstrained formulations. 


\section{CONCLUSION}

The literature on financial optimization models is vast and dates back to the seminal contribution of Markowitz (1952). Four alternative modelling approaches have emerged as suitable frameworks for representing financial optimization problems. They are mean-variance models, discrete-time multi-period models, continuous-time models, and stochastic programming. The mean-variance framework of Markowitz (1952) is widely considered as the starting point for modern research about portfolio optimization. Although this kind of model gets profound insight into the problem, it is of limited use in practice because of two major drawbacks. Firstly, variance is not always a good risk measure for investors; Secondly, a single-period model might be inappropriate for multi-period investment problems with long horizons. Continuous-time models and discrete-time models solved with dynamic programming and optimal control can provide good qualitative insights about fundamental issues in investments and ALM.

The stochastic programming approach for financial optimization can be considered as a practical multi-period extension of the normative investment approach of Markowitz (1952). The advantage of stochastic programming models for multi-period investment and ALM problems is that important practical issues such as transaction costs, multiple state variables, market incompleteness, taxes and trading limits, regulatory restrictions and corporate policy requirements can be handled simultaneously within the framework. Of course this flexibility comes at a price and stochastic programming also has a drawback.

In current application, the methods of output analysis address mainly the two-stage (multi period) stochastic programs. At the same time, Zenious et.al. (1998) validation experiments provide and evidence that even three-stage stochastic programs may outperform significantly the existing static models.

\section{SUGGESTIONS FOR FUTURE WORK}

There are still a lot of interesting topics for further investigations. Some of them are mentioned as follows.

To character the realistic problems, it is very important to set up stochastic programming models that incorporate more consideration of uncertainties. For example, to the portfolio selection problem, in addition to the usual interest rate changes, uncertainty in the timing and amount of cash flows, changes in the default and other risk premia and so on should be considered.

To the ALM, effort should be given to expand the applicability of stochastic programming to address enterprise-wide risk management problems.

When generating scenarios, an important issue is how to measure the approximation error of the returns in the event tree compared to the true underlying distribution. Once appropriate measures have been identified, one could try to develop methods for constructing event trees that minimize the approximation error (assuming the size of the event tree is fixed). A promising first step in this direction is made by Pflug and Swietanowski (1998).

Besides, the present boom of large-scale real-life applications has brought new challenging questions. An important task is an adequate reflection of the dynamic aspects, including further development of tractable numerical approaches. Additional problems are related with the fact that the probability distribution $\mathrm{P}$ is rarely known completely and/or that it has to be approximated for reasons of numerically tractability. The task is to generate the required input, i.e., to approximate $\mathrm{P}$ bearing in mind the required type of the problem ( J.Dupacova, G.Consigli, S.W.Wallace, 2000)

These methods have to be tailored to the structure of the problem and they should also reflect the source, character and precision of the input data.

An extensive research in multistage stochastic programming is an important complex task of the day.

\section{REFERENCES}

Blomvall J, Lindberg PO (2002) A Ricatti-based primal interior point solver for multistage stochastic programming. Eur J Oper Res 143:452-461.

Campbell JY, Chan YL, Viceira LM (2003) A multivariate model of strategic asset allocation. J Financ Econ 67:41-80.

D.R.Carino and W.T.Zeimba. "Formulation of the RussellYasuda Kasai financial planning model.” Operations Research, 46:433-449,1998.

Fleten, Stein-Erik and Lindset, Snorre (2007), "Optimal hedging strategies for multi-period guarantees $n$ the presence of tranasactions costs: A stochastic programming approach”, European Journal of Operational Research, availible online : http://mpra.ub.uni-muenchen.de/7645/.

G.Ch. Pflug and A. Swietanowski. ”Optimal scenario tree generation for multiperiod financial optimization". Technical report Aurora TR1998-22, Vienna University, 1998.

Geyer A, Hanke M, Weissensteiner A (2007) Life-cycle asset allocation and optimal consumption using stochastic linear programming.Working paper, Vienna Graduate School of Finance and University of Innsbruck, available online:

http://papers.ssrn.com/sol3/papers.cfm?abstract_id=952956. 
Geyer A, Ziemba WT (2007) The Innovest Austrian pension fund financial planning model InnoALM. Oper Res 56(4):797-810.

Gondzio J, Grothey A (2007) Solving nonlinear portfolio optimization problems with the primal-dual interior point method. Eur J Oper Res 181:1019-1029.

Gondzio J, Kouwenberg R (2001) High performance computing for asset-liability management. Oper Res 49:879-891.

Gondzio J, Kouwenberg R (2001) High performance computing for asset-liability management. Oper Res 49:879-891.

H.M.Markowitz,”Portfolio selection”. Journal of Finance, 7:77-91, 1952.

J.Dupacova, G.Consigli, S.W.Wallace, "Scenarios for multistage stochastic programs", Annals of Operations Research 100(2000)23-53.

J.R Birge and F. Louveaux, Introduction to Stochastic Programming, Springer-Verlag, New York, 1997.

Li-Y. Yu, X.-D. Ji, and S.-Y. Wang, Stochastic Programming Models in Financial Optimization: A Survey, AMO - Advanced Modeling and Optimization, Volume 5, Number 1, 2003.

Merton RC (1969) Lifetime portfolio selection under uncertainty: the continuous-time case. Rev Econ Stat 51:247-257.

Merton RC (1971) Optimum consumption and portfolio rules in a continuous-time model. J Econ Theory 3:373-413.
P. Brandimarte, Quantitative Methods: An Introduction for Business Management, John Wiley \& Sons, Inc. 2011.

PflugGC, SwietanowskiA(2000) Asset-liability optimization for pension fund management. In: Operations Research Proceedings, Springer, Heidelberg.

Samuelson PA (1969) Lifetime portfolio selection by dynamic stochastic programming. Rev Econ Stat 51:239246.

Wallace SW, Ziemba WT (eds) (2005) Applications of stochastic programming. MPS-SIAM book series on optimization.

Wallace SW, Ziemba WT (eds) (2005) Applications of stochastic programming, MPS-SIAM book series on optimization.

Zenios SA (1999) High-performance computing for financial planning: the last ten years and the next. Parallel Comput 25:2149-2175.

Ziemba, W.T., Mulvey, J.M., eds., Worldwide Asset and Liability modeling. 1998 Cambridge University Press. 